\title{
Flight Performance of a Swashplateless Micro Air Vehicle
}

\author{
James Paulos and Mark Yim
}

\begin{abstract}
We present the control design, system integration, and free flight evaluation of a novel $227 \mathrm{~g}$ swashplateless coaxial helicopter. This micro aerial vehicle (MAV) achieves authority over roll, pitch, and yaw orientation as well as maneuvering thrust using only two propellers directly affixed to two motors. No additional aerodynamic control surfaces or actuators are introduced. Instead, cyclic control is obtained through the underactuated dynamic response of the main rotor itself to a modulated drive torque. Comparisons are drawn to conventional four-motor quadrotors and four-actuator fixed pitch coaxial helicopters in terms of mechanical complexity and actuator mass fraction. Trim power consumption in hover is reported across a range of symmetric and asymmetric loading states. Closed loop trajectory tracking maneuvers are demonstrated in a motion capture environment.
\end{abstract}

\section{INTRODUCTION}

Small micro air vehicles (MAV) are becoming popular, particularly rotorcraft with the ability to hover and position arbitrarily in 3D space. These capabilities support a wide range of applications from the inspection of hazardous environments, to package delivery, to toys. Such MAV come in a variety of forms including single rotor helicopters with tail rotors, coaxial rotor helicopters with two counter-rotating blades on a shared vertical axis, and quadrotors incorporating four lifting rotors. Of these, the quadrotor has received much recent interest owing to its simplicity of control [1] and relatively simple mechanical design.

Making flying vehicles smaller, simpler, and lower cost is the goal of this work. Reducing the number of actuators can promote these goals as long as complicated mechanisms are not also added in their place. Almost all MAVs are considered under-actuated as they incorporate fewer than six actuators to control the six degrees of freedom of the vehicle position and orientation [2]. Many have four actuators with a mapping to attitude torques (roll, pitch, yaw) and a thrust force. Quadrotors by definition have four actuators for the four rotors. Fixed collective helicopters have the main lift rotor, a tail rotor, and two servomotors which affect the main rotor pitch through a swashplate. Even tri-rotor craft typically augment their three propeller drives with an additional fourth actuator to adjust the pitch angle of one propeller [3].

We propose a MAV with control of attitude and lift, but with just two actuators driving coaxial rotors. A simple passive hinged rotor responds to pulsed torques to achieve swashplate-like attitude authority. In a sense, the mechanical complexity of swashplate linkages and servomotors is replaced by low level computation and electronic drive of

The authors are with the Dept. of Mech. Eng. \& Appl. Mechanics and GRASP Lab at the University of Pennsylvania, Philadelphia, PA. jpauloseseas. upenn. edu, yimegrasp.upenn. edu the cyclic motor torque. In addition, the vehicle dynamics remain amenable to stabilization with common quadrotorlike controllers using an appropriate mapping of inputs.

\section{RELATED WORK}

Single rotor helicopters and coaxial helicopters use the main lifting rotor(s) both to generate thrust for flight and to regulate the vehicle's orientation in roll and pitch. This is conventionally achieved through a technique referred to as cyclic control in which the pitch of the rotor blades is manipulated within each revolution. For example, the airfoils might elevate their pitch while passing across the nose of the aircraft and depress their pitch while passing across the tail of the aircraft, resulting in an asymmetry of lift across the effective rotor disk. This manifests as a direct moment applied to the vehicle as well as a tilting of the effective tip path plane, associated with a slight vectoring of thrust. Cyclic control is typically achieved by adding two or more servomotors near the hub which actuate the blade pitch axis through the action of several linkages and a swashplate as in Fig. 11.

The mechanical complexity of this solution and the difficulty of its realization at small scale in MAV have motivated a variety of innovations. Piezoelectric actuators have been used in place of servomotors to drive a conventional swashplate [4], the motor orientation itself has been adjusted in flight by piezoelectric actuators [5], and shape memory alloys have been used to deform the gross airframe supporting the rotor assembly [6]. In other work, control is achieved by adjusting the vehicle center of mass in flight [7] or introducing additional outboard propellers [8]. In all but the last of these systems, a conventional passive stabilizer bar mechanism similar to Fig. 10 was also required in order to augment the active control authority.

\section{OPERATING PRINCIPLE}

In this work we directly induce a controlled cyclic response in blade pitch by modulating the main drive motor already present. Such a strategy is described in [9], where a propeller assembly with explicit pitch hinges is driven indirectly through an offset flexible linkage. The alternative direct method favored here was first suggested by the authors in [10], where static test stand experiments are discussed.

As shown in Fig. 1, two blades are attached to the rotor hub by simple pin hinges at a small radius from the center of rotation. Crucially, the lines of these hinges are not vertical, as is typically found in the lead-lag hinges of a conventional helicopter. Instead, the top of the "positive" blade's hinge is inclined inward, and the top of the "negative" blade's hinge is 


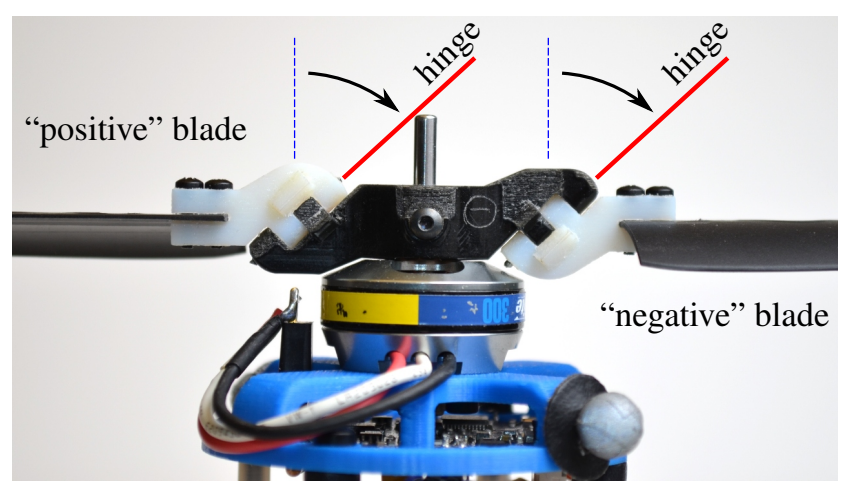

Fig. 1. Two blades are attached by canted hinges to a hub directly affixed to the main motor
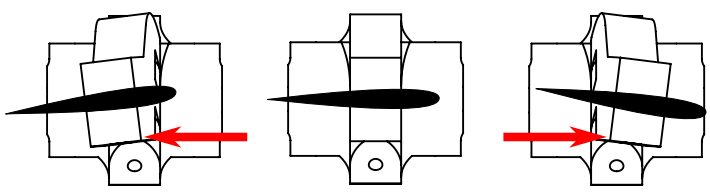

Fig. 2. As the positive blade lags backwards, the pitch increases. As it leads forward, the pitch decreases.

inclined outward. This geometry couples the lead-lag motion of the blade tip about the central shaft to a pitching motion about the blade long axis, as depicted in Fig. 2 .

The objective of the cyclic system is to induce an elevated blade pitch as the blades pass some station of the rotor disk and a depressed blade pitch as the blades pass $180^{\circ}$ opposite. To this end, we modulate the torque applied to the motor by adding a sinusoidal component in phase with the motor rotation, exciting once per revolution variations in lag angle and therefore also in pitch. As the hub accelerates forwards the positive blade tip lags backwards relative to the hub and the kinematics require the pitch of the blade tip to increase. At the opposite station, $180^{\circ}$ later, the positive blade tip now leads forwards relative to the hub and the pitch of the blade is instead depressed. The complementary geometry of the negative blade yields the opposite response, so that an appropriate input can induce both blades to, for example, elevate pitch while passing across the nose and decrease pitch while passing across the tail of the aircraft. Such smooth oscillation through every revolution bears a strong resemblance to conventional cyclic pitch control, but it is now achieved merely by electronically altering the amplitude and phase offset angle of the sinusoidal drive component.

\section{SySTEM INTEGRATION}

\section{A. Mechanical Design}

The novel cyclic control method permits a very simple mechanical design for the complete MAV, as shown in Fig. 3 . The vehicle has a $30.0 \mathrm{~cm}$ diameter top rotor, stands $16.3 \mathrm{~cm}$ tall, and has a flying weight of $227 \mathrm{~g}$. It is comparable in scale to the conventional coaxial Blade CX2 used in [11], [12] which has a $34.5 \mathrm{~cm}$ rotor diameter, a height of $18.4 \mathrm{~cm}$, and an identical flying weight of $227 \mathrm{~g}$.

The hinged control rotor is directly mounted to the top motor, pointed upwards. A rigid rotor is mounted to the

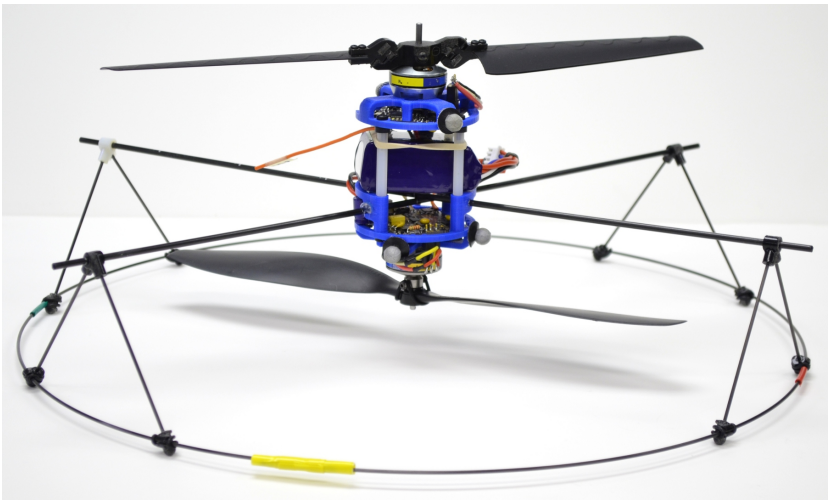

Fig. 3. A $227 \mathrm{~g}$ coaxial MAV exhibits cyclic control without a swashplate or any additional actuators.

bottom motor, which is inverted to place the rotor at the bottom of the vehicle. This arrangement has been used in the past to avoid the complexities of hollow shaft, concentric drive systems [4], [5]. A bottom rotor guard ring and landing gear supports the vehicle, enabling free takeoff and landing. While this configuration is expedient, the bottom rotor might also be replaced by a tail rotor or a second top rotor in order to permit an underslung payload.

\section{B. Electrical Design}

A pair of identical custom avionics boards mounted at the rear of each motor support communication, inertial sensing, computation, and motor control. Onboard controllers are executed by a 32 bit microcontroller (STM32f373) running at $72 \mathrm{MHz}$. A $900 \mathrm{MHz}$ low power transceiver (AT86RF212) enables half duplex communication at $250 \mathrm{kbits} / \mathrm{s}$ for command reception and transmission of flight data.

A hall effect encoder on the circuit board detects the orientation of a diametrically polarized magnet affixed to the bottom of the spinning motor shaft. This absolute position information is used to generate the three phase drive for the brushless motor, enabling both conventional steady commutation to spin the motor and synthesizing our once-per-cycle applied sinusoidal drive component.

An onboard inertial measurement unit (MPU6050 IMU) provides acceleration and rotation rate measurements at $250 \mathrm{~Hz}$ to the onboard attitude estimator and controller. Finally, onboard voltage and current sensing enables precise power measurements during maneuvers for offline evaluation. Presently only the top avionics board executes the flight controllers and state estimator, and the bottom avionics board acts as a simple I2C slave motor controller.

\section{Control Rates and Timescales}

The timescales and update rates of the cascaded communications and control system are summarized in Fig. 4. During maneuvers, thrust and desired attitude commands are received at $75 \mathrm{~Hz}$. These may be direct commands from a human pilot, or autonomously generated in the motion capture environment. The onboard attitude controller updates at $250 \mathrm{~Hz}$, generating motor commands expressed as a mean value, amplitude, and phase offset parameters. Output to 


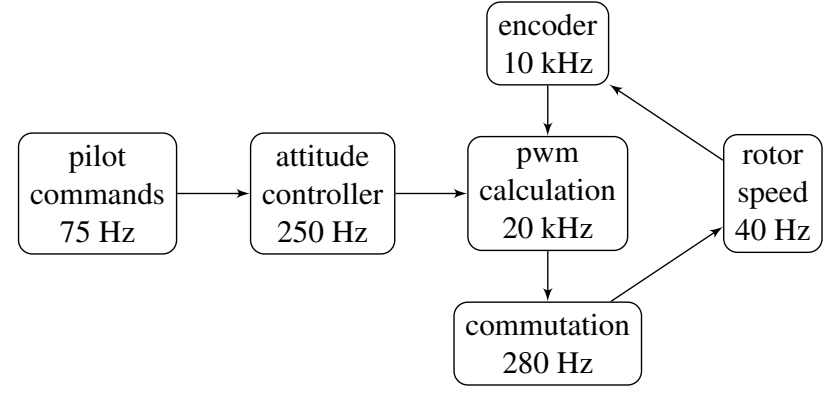

Fig. 4. Representative frequencies. The attitude control rate is decoupled from both the rotor speed and pwm synthesis of the pulsing torque.

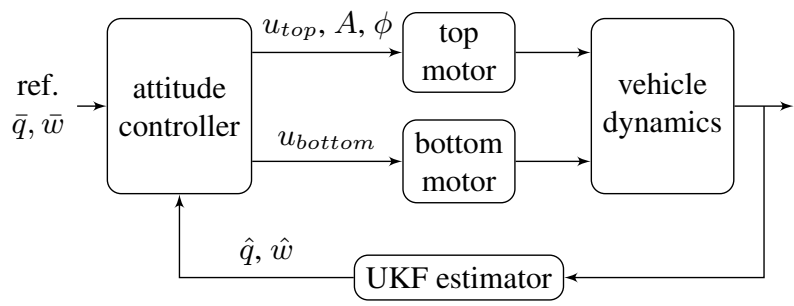

Fig. 5. The controller operates on an estimated orientation and angular rate $(\hat{q}, \hat{w})$ and desired values $(\bar{q}, \bar{w})$. Outputs are mean drive voltages $u_{\text {bottom }}$, $u_{\text {top }}$ and an additive sinusoidal component of amplitude $A$ and phase $\phi$.

the motor is based on these parameters and the motor's instantaneous mechanical orientation.

The sinusoidal component of the motor control needs to be synthesized smoothly even as the propeller spins at approximately $40 \mathrm{~Hz}$. At this speed, the normal three phase electrical commutation frequency is approximately $280 \mathrm{~Hz}$ (the motor has seven pole pairs). In comparison, synthesis of the $40 \mathrm{~Hz}$ superimposed sinusoid we require does not represent an unusual burden. The motor outputs are updated at the $20 \mathrm{kHz}$ pulse width modulation frequency based on position estimates from the absolute encoder, which updates internally at $10 \mathrm{kHz}$. The driver circuitry and the motor itself are completely conventional. The critical aspect of these control layers is that the generation of the pulsing torque which enables cyclic control is abstracted from the attitude controller as mean value, amplitude, and phase parameters. This strongly decouples the attitude controller rate from either the propeller speed or the motor drive update rate.

\section{Attitude Control}

Automated trajectory tracking is achieved with a nested inner attitude controller and outer position and velocity tracking controller. This follows the common approach of cascading controllers for trajectory tracking [1] or path following [12] tasks. The inner attitude controller onboard the vehicle is depicted in Fig. 5.

Onboard the vehicle, an unscented Kalman filter following [13] forms an estimated orientation quaternion $\hat{\boldsymbol{q}}$ and angular velocity vector $\hat{\boldsymbol{w}}$ based on measurements from the onboard IMU. Our controller employs a nonlinear attitude tracking controller operating directly on quaternions as in [14], which is widely practiced. A desired body moment vector $\boldsymbol{u}$ is computed from the estimates $\hat{\boldsymbol{q}}, \hat{\boldsymbol{w}}$ and the desired orientation and angular velocities $\overline{\boldsymbol{q}}, \overline{\boldsymbol{w}}$ based on an orientation error vector $\boldsymbol{e}_{\boldsymbol{R}}$ and angular rate error vector $\boldsymbol{e}_{\boldsymbol{w}}$ with diagonal gain matrices $K_{R}$ and $K_{w}$.

$$
\boldsymbol{u}=-K_{R} \boldsymbol{e}_{\boldsymbol{R}}-K_{w} \boldsymbol{e}_{\boldsymbol{w}}
$$

The angular rate error vector is intuitively defined.

$$
e_{w}=\hat{w}-\bar{w}
$$

To form the orientation error vector $e_{\boldsymbol{R}}$, we consider the error quaternion $\boldsymbol{q}_{\boldsymbol{e}}=\overline{\boldsymbol{q}}^{*} \hat{\boldsymbol{q}}$ whose real and scalor parts $\boldsymbol{q}_{\boldsymbol{e}}=$ $(\cos (\theta / 2), \boldsymbol{v} \sin (\theta / 2))$ describe a rotation of $\theta$ radians about a unit vector $\boldsymbol{v}$. We define the error vector $\boldsymbol{e}_{\boldsymbol{R}}=\sin (\theta / 2) \boldsymbol{v}$ by extracting the vector part of the error quaternion.

$$
\boldsymbol{e}_{\boldsymbol{R}}=\operatorname{sign}(s) \boldsymbol{x}, \text { given } \boldsymbol{q}_{\boldsymbol{e}}=(s, \boldsymbol{x})
$$

As noted in [15], this bears a resemblance to an alternative matrix formulation suggested in [16].

In the new vehicle, the calculated control vector $\boldsymbol{u}$ and a commanded thrust voltage $f$ determine the top rotor mean drive voltage $u_{\text {top }}$, bottom rotor mean drive $u_{\text {bottom }}$, top rotor pulsing amplitude $A$, and top rotor pulsing phase $\phi$. Collective increase and decrease of $u_{\text {bottom }}$ and $u_{t o p}$ increases and decreases net thrust. A differential between $u_{\text {bottom }}$ and $u_{\text {top }}$ affects a differential torque between the counter rotating propellers and yaws the vehicle. Pitch and roll corrections are achieved through the sinusoid amplitude $A$ and phase angle $\phi$ which are determined by the magnitude and direction of the desired in plane control moment vector $\left\{u_{x}, u_{y}\right\}$. In practice the amplitude $A$ is modified to eliminate a deadband value $A_{0}$ below which no cyclic oscillation is excited.

$$
\begin{aligned}
u_{\text {bottom }} & =f+u_{z} \\
u_{\text {top }} & =f-u_{z} \\
A & =\sqrt{u_{x}^{2}+u_{y}^{2}}+A_{0} \\
\phi & =\operatorname{atan} 2\left(u_{y}, u_{x}\right)
\end{aligned}
$$

This very simple mapping from $\{f, \boldsymbol{u}\}$ to $\left\{u_{\text {bottom }}, u_{\text {top }}\right.$, $A, \phi\}$ achieves adequate performance near trim. A fixed calibration offset $\theta_{0}$ relates the blade attachment angle to the rotation sensor and airframe. The final applied voltages are based on the instantaneously measured rotor position $\theta$.

$$
\begin{aligned}
V_{\text {bottom }} & =u_{\text {bottom }} \\
V_{\text {top }} & =u_{\text {top }}+A \cos \left(\theta-\theta_{0}-\phi\right)
\end{aligned}
$$

\section{POWER FOR HOVER}

\section{A. Symmetric Loading}

Many useful tasks call for a MAV to loiter near a site of interest while supporting a sensor payload. The impact of added weight on power consumption was investigated by hovering with added masses of up to $18 \%$ of the vehicle weight. Power consumption was measured onboard in flight, representing the combined requirements of the hosted electronics, thrust, and attitude control action. The rise in power consumption and rise in rotor speeds are shown in Fig. 6 and 7 for trials at five distinct loads. Importantly, the pulsing 
Power in Hover, Various Payloads

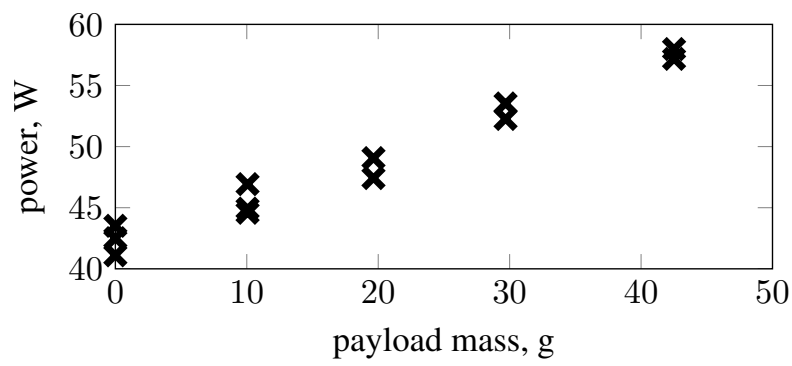

Fig. 6. Electrical power consumption in hover with various payload masses.

Rotor Speed in Hover, Various Payloads

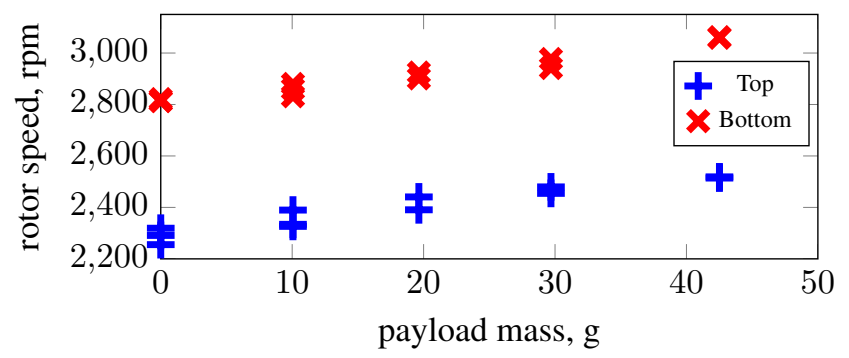

Fig. 7. Rotor speed of the top (pulsing) and bottom (non-pulsing) propellers in hover with various payload masses.

cyclic strategy works satisfactorily across the range of rotor speeds, requiring no parameter tuning.

These tests also establish that very little power in hover is wasted due to the pulsing attitude stabilization action. Roll and pitch control was disabled for brief periods of $0.5 \mathrm{~s}$ or more during which power consumption was monitored after waiting $0.25 \mathrm{~s}$ for initial transients to abate. This test accurately captures the inflow condition and torque balance between the propellers in flight. We also observe that pulsing control does not notably affect rotor speed in the hover condition. Over ten trials the mean power consumption was $40.6 \mathrm{~W}$ with a standard deviation of $1.0 \mathrm{~W}$. As expected, little control effort is exerted in the hover condition and the hover power consumption of $42.4 \mathrm{~W}$ does not greatly exceed the thrust requirements alone.

For comparison, the addition of just the $24 \mathrm{~g}$ in dead mass representing the servos, linkages, and stabilizer bar of the comparable CX2 helicopter would increase the hover power requirement by more than $5.9 \mathrm{~W}$ to $114 \%$ of the original. The removal of these components and their replacement with pulsing cyclic control represents an improvement in projected hover endurance, even neglecting the power requirements of the discarded actuators themselves.

For a fixed thrust, momentum theory indicates that increasing rotor area strongly correlates with decreased power requirements. This has been cited as a possibly advantage of large single rotor craft over conventional quadrotors, an advantage which motivates the hybrid vehicle in [8]. Our method of cyclic control is another approach towards aerodynamically efficient rotorcraft which dispatch with the weight and complexity of servomotors, linkages, and swashplates.
Power in Hover, $10 \mathrm{~g}$ Asymmetric Loads

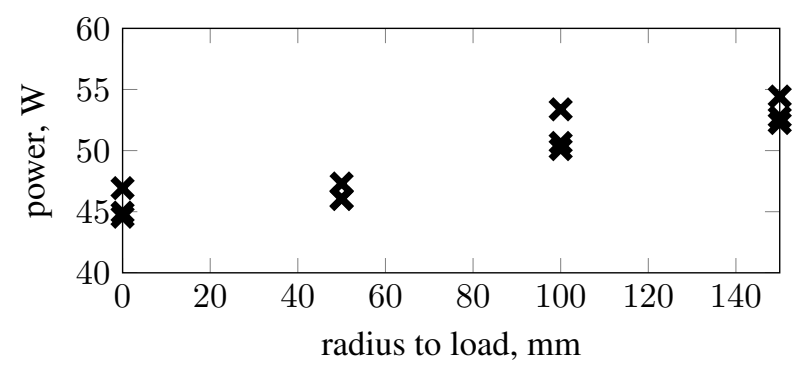

Fig. 8. Electrical power consumption in hover with a $10 \mathrm{~g}$ payload offset laterally from the vehicle center.

Rotor Speed in Hover, $10 \mathrm{~g}$ Asymmetric Loads

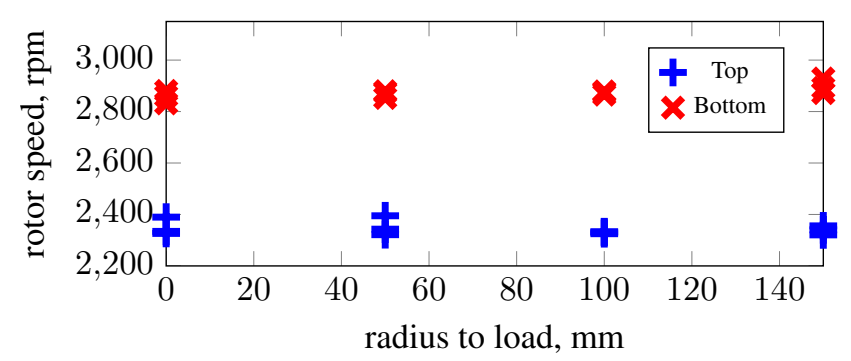

Fig. 9. Rotor speed of the top (pulsing) and bottom (non-pulsing) propellers in hover with a $10 \mathrm{~g}$ payload offset laterally from the vehicle center.

\section{B. Assymmetric Loading}

In many cases a MAV can be co-designed with its payload to control the center of mass placement throughout the operation. For example, a fixed camera or a deployable chemical sensor might be considered in the initial design. However, an airframe retrofit or collection of an unknown payload can result in an asymmetric loading that must be balanced in flight by the vehicle's attitude control authority.

A series of hover tests demonstrate the ability of the pulsing blade control to overcome such persistent disturbances. Fig. 8 shows the increase in power requirements as a $10 \mathrm{~g}$ payload is repositioned from the center out towards the periphery of the vehicle. As expected, the rotor speeds remain relatively constant throughout these tests (Fig. 9) and the significant increase in power is due to the active pulsing control needed to maintain level flight. As with all MAVs, the location of the center of mass remains an important constraint for vehicles incorporating this style of attitude control.

\section{ACtuator Mass Budget}

After the battery itself, actuator mass represents the second largest fraction of vehicle weight in five of the six quadrotors surveyed in [17], an ensemble of micro air vehicles from $43 \mathrm{~g}$ to $967 \mathrm{~g}$. Conventional coaxial helicopters like the CX2 require only two drive motors instead of four, but must integrate a swashplate mechanism (Fig. 11), additional servomotors to drive it, and potentially a passive stabilizer bar to reduce the required control bandwidth (Fig. 10). Fig. 12 compares the mass budget of the $227 \mathrm{~g}$ pulsing coaxial helicopter to the $227 \mathrm{~g}$ conventional CX2 coaxial helicopter and an ensemble average quadrotor distribution from [17]. 


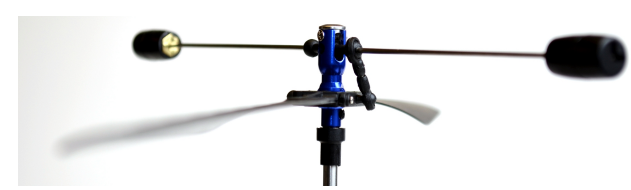

Fig. 10. Conventional helicopters often incorporate a passive stabilizer bar mechanism to augment active control.

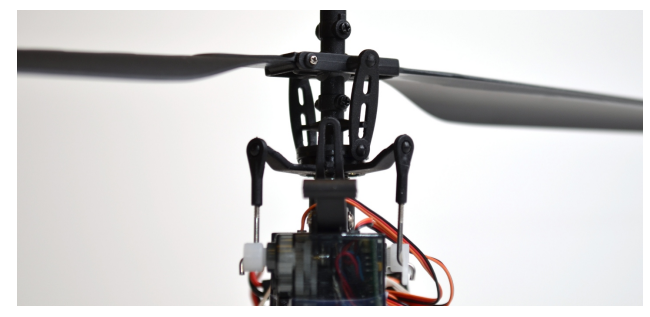

Fig. 11. This conventional system incorporates two servomotors at bottom with linkages upwards to a swashplate, and additional linkages upward to actuate the blade pitch axis. Six ball and socket joints are required.

Elimination of the swashplate and actuators represents a significant reduction in the total actuator mass, therefore allowing larger onboard batteries and potentially longer flight endurance. A complete breakdown of the mass distribution within the pulsing coaxial helicopter is provided in Table I.

\section{MANUfaCtURABILITY AND COST}

The rapid growth of quadrotor use in the MAV space owes in part to their mechanical simplicity and ease of manufacture. For quadrotors, the only moving parts are the commercial off-the-shelf motors themselves. Emerging manufacturing methods such as 3D printing, origami inspired folding, and laser cut fabrication have all been applied to rigid airframes [18], enabling prototyping and small batch production with low cost equipment.

In contrast, the conventional swashplate control system of the CX2 involves eight distinct ball and socket joints attending to the five linkages shown in Figs. 10 and 11 in addition to a ball bearing for the swashplate itself and free feather hinges for each blade. These types of precision components are a barrier to affordable direct additive prototyping. Further, while they are readily produced by injection molding in a factory environment, they introduce a laborious final assembly procedure. If fouled by grit from the environment,

\section{Subsystem Mass Fraction}

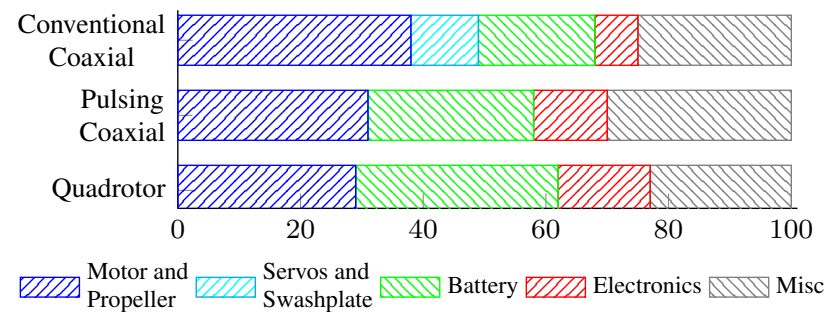

Fig. 12. The swashplate and servomotors are a large fraction of the mass in a conventional coaxial helicopter (Blade CX2) but are eliminated in a pulsing coaxial helicopter and in quadrotors (ensemble averages from [17]).
TABLE I

Pulsing CoAxial MAV Component Masses

\begin{tabular}{rr}
\hline component & mass \\
\hline hinged propeller and fasteners & $11.3 \mathrm{~g}$ \\
fixed propeller and fasteners & $10.2 \mathrm{~g}$ \\
brushless motors & $2 \times 24.5 \mathrm{~g}$ \\
control circuit boards & $2 \times 14.0 \mathrm{~g}$ \\
$11.1 \mathrm{~V}, 850 \mathrm{mAh}$ battery & $76.1 \mathrm{~g}$ \\
landing gear & $18.3 \mathrm{~g}$ \\
$8 \mathrm{~mm}$ reflective markers (4) & $2.6 \mathrm{~g}$ \\
airframe and misc. hardware & $31.6 \mathrm{~g}$ \\
\hline total & $227 . \mathrm{g}$
\end{tabular}

they will require field maintenance. The associated actuator cost is also not insignificant: the two $\$ 15$ servos in a CX2 actually cost more than the two $\$ 10$ brushed drive motors.

Direct cyclic control through pulsing torques introduces only two simple pin hinges and completely eliminates the need for ball and socket joints or ball bearings. The hinged hub itself is readily manufactured on common 3D printers, and could be molded directly as a feature of the propeller in a large scale operation. As a result, halving the number of motors, motor controllers, and propellers as found in a quadrotor can in fact reduce the overall component count and assembly time for these types of micro air vehicles.

\section{Acoustics}

The adoption of this control strategy may confer other indirect benefits which require further investigation. Anecdotally, colleagues more familiar with quadrotors often comment on how quiet the coaxial vehicle is in flight. The central large rotors are much slower than the small rotors of a similarly sized quadrotor such as the KMel Nano+, which may help reduce noise levels. The median sound level in hover is $62 \mathrm{dBA}$ for the pulsing coaxial helicopter and $70 \mathrm{dBA}$ for the quadrotor, ${ }^{1}$ a perceptually significant difference. Simultaneously, we have eliminated the servomotors, stabilizer bar, and gearbox found in most helicopters, each a potential source of noise. Future work may consider how to leverage this design to promote quiet operation, improving user confidence in close quarters and opening new applications and operational environments to MAVs.

\section{TRajectory TRACKing}

The two motor system is capable of tracking 3D reference trajectories through space while independently regulating the heading orientation, making these vehicles suitable for a variety of camera pointing or precision delivery and retrieval tasks. In this respect the cyclic system maintains the advantage of quadrotors and swashplate systems over the many fixed wing, ornithopter, and toy helicopter systems which can not reject lateral positioning disturbances without turning.

A $20 \mathrm{~cm}$ lateral step in desired position excites a roll response and position correction as shown in Fig. 13. During this brief maneuver the vehicle attains a maximum roll angle of $9^{\circ}$ and speed of $0.47 \mathrm{~m} / \mathrm{s}$. Such a side step motion resembles the attitude controller response to a lateral double shown in [12] for a similar scale conventional coaxial helicopter.

\footnotetext{
${ }^{1}$ As measured at $1 \mathrm{~m}$ distance by a consumer grade sound level meter.
} 
Response to Lateral Position Step
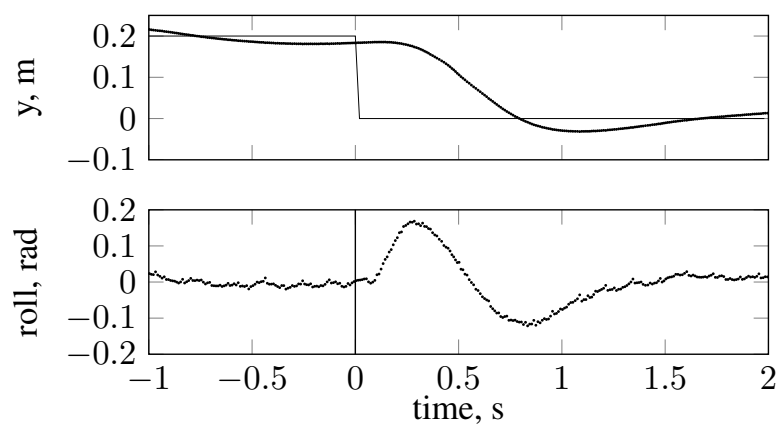

Fig. 13. A $20 \mathrm{~cm}$ step in desired position towards the right results in a roll response and corrective lateral motion.

\section{D Trajectory Tracking}

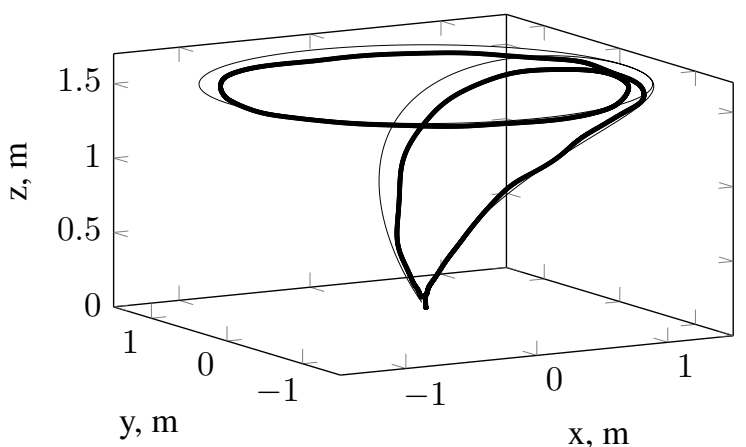

Fig. 14. Automated tracking of a spiral ascent to $1.5 \mathrm{~m}$ height, a $1.5 \mathrm{~m}$ radius circuit at $1 \mathrm{~m} / \mathrm{s}$, and return to origin.

Fig. 14 shows a more realistic multi-axis maneuver in which the vehicle executes a spiral ascent up to $1.5 \mathrm{~m}$ altitude, completes one circuit of a $1.5 \mathrm{~m}$ radius circle at $1 \mathrm{~m} / \mathrm{s}$ cruising speed, and finally descends to land at the origin. Throughout the maneuver the vehicle maintains a northward heading, demonstrating independent control over both direction of travel and the yaw orientation. Free flight maneuvers are demonstrated in the video supplement.

\section{Conclusions}

We have presented the design, control strategy, and first flight performance results of a swashplateless coaxial helicopter. This device is capable of the trajectory tracking and heading control maneuvers expected from quadrotors and helicopters, but accomplishes these control tasks using only two onboard actuators and not the conventional four or five found in typical MAVs. While only a prototype, this vehicle already has the capabilities required to pursue a number of contemporary research questions in vision and control currently being supported by the conventional technology.

Cost, complexity, and reliability will continue to be fundamental challenges in real world civilian applications of MAV technology. This design reduces the number of expensive motor and power electronics components required when compared to quadrotor or conventional helicopter platforms. It also eliminates the demanding final assembly and maintenance issues associated with the swashplate systems found in most helicopters. Such simple, affordable, and robust vehicles will assist in making micro air vehicles a commodity technology available to nonspecialists for real world use. In future work we intend to combine our method of cyclic control from a single actuator with work in passively stable spinning body rotorcraft [19] to create new extremely simple and scalable micro air vehicles.

\section{ACKNOWLEDGEMENT}

The authors wish to thank Matthew Piccoli for development of the avionics hardware used in these flight tests.

\section{REFERENCES}

[1] D. Mellinger and V. Kumar, "Minimum snap trajectory generation and control for quadrotors," in Robotics and Automation (ICRA), 2011 IEEE International Conference on, May 2011, pp. 2520-2525.

[2] F. Kendoul, "Survey of advances in guidance, navigation, and control of unmanned rotorcraft systems," Journal of Field Robotics, vol. 29, no. 2, pp. 315-378, 2012.

[3] S. Salazar-Cruz, R. Lozano, and J. Escareño, "Stabilization and nonlinear control for a novel trirotor mini-aircraft," Control Engineering Practice, vol. 17, no. 8, pp. 886-894, 2009.

[4] D. Schafroth, S. Bouabdallah, C. Bermes, and R. Siegwart, "From the test benches to the first prototype of the mufly micro helicopter," $J$. of Intelligent and Robotic Syst., vol. 54, no. 1-3, pp. 245-260, 2009.

[5] S. George and P. Samuel, "On the design and development of a coaxial nano rotorcraft," in 50th AIAA Aerospace Sciences Meeting. American Institute of Aeronautics and Astronautics, Jan. 2012.

[6] P. Samuel, J. Sirohi, F. Bohorquez, and R. Couch, "Design and testing of a rotary wing MAV with an active structure for stability and control," in Proceedings of the AHS 61st Annual Forum. American Helicopter Society, 2005.

[7] C. Bermes, S. Leutenegger, S. Bouabdallah, D. Schafroth, and R. Siegwart, "New design of the steering mechanism for a mini coaxial helicopter," in Intelligent Robots and Systems (IROS), 2008 IEEE/RSJ International Conference on, Sept 2008, pp. 1236-1241.

[8] S. Driessens and P. Pounds, "Towards a more efficient quadrotor configuration," in Intelligent Robots and Systems (IROS), 2013 IEEE/RSJ International Conference on, Nov 2013, pp. 1386-1392.

[9] Y. Fujihira, R. Sasaki, and M. Ando, "Propeller blade tip path plane inclining device," U.S. Patent 5259729 , November, 1993.

[10] J. Paulos and M. Yim, "An underactuated propeller for attitude control in micro air vehicles," in Intelligent Robots and Systems (IROS), 2013 IEEE/RSJ International Conference on, Nov 2013, pp. 1374-1379.

[11] S. Han, A. Straw, M. Dickinson, and R. Murray, "A real-time helicopter testbed for insect-inspired visual flight control," in Robotics and Automation (ICRA), 2009 IEEE International Conference on, May 2009, pp. 3055-3060.

[12] N. Dadkhah and B. Mettler, "Control system design and evaluation for robust autonomous rotorcraft guidance," Control Engineering Practice, vol. 21, no. 11, pp. 1488 - 1506, 2013.

[13] E. Kraft, "A quaternion-based unscented kalman filter for orientation tracking," in Proceedings of the Sixth International Conference of Information Fusion, vol. 1, July 2003, pp. 47-54.

[14] B. Wie and P. M. Barba, "Quaternion feedback for spacecraft large angle maneuvers," Journal of Guidance, Control, and Dynamics, vol. 8 , no. 3, pp. $360-365,1985$.

[15] V. Kumar and N. Michael, "Opportunities and challenges with autonomous micro aerial vehicles," The International Journal of Robotics Research, vol. 31, no. 11, pp. 1279-1291, 2012.

[16] T. Lee, M. Leoky, and N. H. McClamroch, "Geometric tracking control of a quadrotor UAV on SE(3)," in Decision and Control (CDC), 2010 49th IEEE Conference on, 2010, pp. 5420-5425.

[17] Y. Mulgaonkar, M. Whitzer, B. Morgan, C. M. Kroninger, A. M. Harrington, and V. Kumar, "Power and weight considerations in small, agile quadrotors," in Proc. SPIE, vol. 9083, 2014.

[18] A. M. Mehta, D. Rus, K. Mohta, Y. Mulgaonkar, M. Piccoli, and V. Kumar, "A scripted printable quadrotor: Rapid design and fabrication of a folded MAV," in Robotics Research (ISRR), 16th Int. Symp. of, December 2013.

[19] M. Piccoli and M. Yim, "Passive stability of a single actuator micro aerial vehicle," in Robotics and Automation (ICRA), 2014 IEEE/RSJ International Conference on, June 2014. 